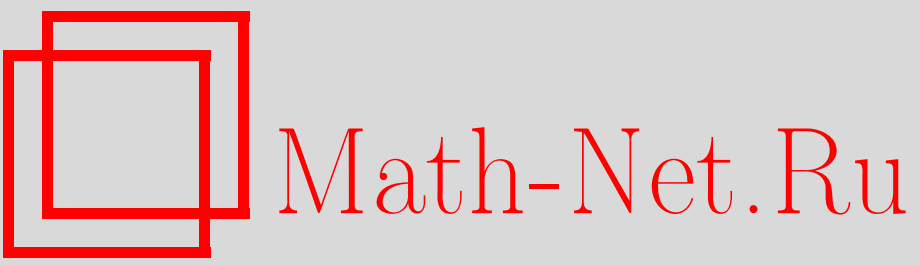

В. В. Веденяпин, С. А. Амосов, Л. Тоскано, Инварианты для гамильтонианов и кинетических уравнений, УМН, 1999, том 54, выпуск 5, 153-154

DOI: https://doi.org/10.4213/rm223

Использование Общероссийского математического портала Math-Net.Ru подразумевает, что вы прочитали и согласны с пользовательским соглашением

http://www . mathnet.ru/rus/agreement

Параметры загрузки:

IP : 34.239 .49 .27

26 апреля 2023 г., 09:38:53 


\section{ИНВАРИАНТЫ ДЛЯ ГАМИЛЬТОНИАНОВ И КИНЕТИЧЕСКИХ УРАВНЕНИЙ}

В. В. ВедЕняпин, С. А. Амосов, Л. Тоскано

1. Рассмотрим полиномиальные гамильтонианы $H$ в переменных $z_{k}=\left(x_{k}+i p_{k}\right) / \sqrt{2}$, $\bar{z}_{k}=\left(x_{k}-i p_{k}\right) / \sqrt{2}, k=1, \ldots, N: H=\sum_{(\alpha, \beta) \in J} b_{\alpha \beta}\left(z^{\alpha} \bar{z}^{\beta}+\bar{z}^{\alpha} z^{\beta}\right)$.

Здесь $\alpha, \beta \in \mathbb{Z}_{+}^{N}$ - целочисленные векторы с неотрицательными компонентами, $J$ есть некоторый набор пар таких векторов. Им соответствуют квантовые гамильтонианы

$$
H=\sum_{(\alpha, \beta) \in J} b_{\alpha \beta}\left(a^{\alpha} a^{+\beta}+a^{+^{\alpha}} a^{\beta}\right) .
$$

Здесь $a^{\alpha}=a_{1}^{\alpha_{1}} \cdots a_{N}^{\alpha_{N}}$, и $a_{i}^{+}$и $a_{i}$ есть операторы рождения и уничтожения с бозонными или фермионными соотношениями коммутации [1]. В [2], [3] было доказано, что $I=\sum_{i=1}^{N} r_{i} a_{i}^{+} a_{i}$ есть инвариант для (1), т.е. $[I, H]=0$, если $(\mathbf{r}, \alpha-\beta)=0$ для всех $(\alpha, \beta) \in J$.

Сопоставим каждому гамильтониану (1) систему уравнений в частных производных на функции $f_{i}(t, x), x \in \mathbb{R}^{d}$, вида

$$
\frac{\partial f_{i}}{\partial t}+\left(\nu_{i}, \frac{\partial f_{i}}{\partial x}\right)=\sum_{(\alpha, \beta) \in J}\left(\alpha_{i}-\beta_{i}\right) B_{\alpha \beta}\left(h^{\beta}-h^{\alpha}\right) .
$$

Здесь $h^{\alpha}=h_{1}^{\alpha_{1}} \cdots h_{N}^{\alpha_{N}}, h_{i}=f_{i} /\left(1+\theta_{i} f_{i}\right), \theta_{i}=1$ для бозонов, $\theta_{i}=-1$ для фермионов, $\theta_{i}=0$ в случае классической статистики Больцмана, $B_{\alpha \beta}=\sigma_{\beta}^{\alpha}\left(1+\theta_{1} f_{1}\right)^{\alpha_{1}+\beta_{1}} \ldots$ $\left(1+\theta_{N} f_{N}\right)^{\alpha_{N}+\beta_{N}}$, где $\sigma_{\beta}^{\alpha}>0$ - сечение реакций (столкновений), $\nu_{i} \in \mathbb{R}^{d}-$ скорости движения $i$-го вещества. Уравнения (2) обобщают дискретные модели уравнения Больцмана и Улинга-Уленбека [4], [5] и имеют аналогичные законы сохранения: $I=\sum_{i=1}^{N} \int_{\mathbb{R}} \mu_{i} f_{i}(t, x) d x$ есть инвариант для $(2)$, если $(\mu, \alpha-\beta)=0$ для всех $(\alpha, \beta) \in J$. Система (2) удовлетворяет $H$-теореме: $H$-функция $H=\sum_{i=1}^{N} H_{\theta_{i}}\left(f_{i}\right)$ убывает в силу $(2)$, если $H_{\theta}(f)=f \ln f-\theta(1+\theta f) \ln (1+\theta f)$.

2. Уравнение (2) называется дискретной моделью по скоростям (ДМС) уравнения Больцмана, если: а) $\alpha=\mathbf{e}_{i}+\mathbf{e}_{j}, \beta=\mathbf{e}_{k}+\mathbf{e}_{l}$, где $\mathbf{e}_{k}-$ вектор стандартного базиса в $\mathbb{Z}_{+}^{N}$ с 1 на $k$-м месте и нулями на остальных; при этом все четыре индекса $(i, j, k, l)$ различны: это соответствует парному столкновению $(i, j) \leftrightarrow(k, l)$ частиц; б) $\nu_{i}$ и $\sigma_{\beta}^{\alpha}$ выбраны так, чтобы выполнялись законы сохранения импульса, энергии и числа частиц.

Если имеется $r$ сортов частиц с массами $m_{1}, \ldots, m_{r}$, то дискретная модель $(2)$ должна иметь $r+d+1$ инвариантов, а именно, $r$ интегралов числа частиц каждого сорта (если нет превращений масс в реакциях $), d$ компонент суммарного импульса и один интеграл энергии (если возможны только упругие столкновения).

До недавнего времени не было известно при $r>1$ ни одной нетривиальной ДМС (cм. [6]) с правильным числом инвариантов. Под нетривиальными авторы [6] понимают модели с обменом энергией, а предложенные в [6] две нетривиальные модели обладают, как показано в [7], одним и двумя лишними инвариантами соответственно. Модели без лишних инвариантов названы в [6] нормальными.

Построение моделей с нужным числом инвариантов проводится в [7] индукцией по числу $N$ с подсчетом инвариантов на каждом шаге. Следующая модель для одномерного случая является рекордной: число скоростей $N$ в ней минимально в классе нормальных нетривиальных моделей, симметричных относительно нуля.

Работа выполнена при частичной поддержке Российского фонда фундаментальных исследований (грант № 98-01-00861). 
Модель 1 (С.А. Амосов, В.В. Веденяпин). $d=1, r=2, m_{1} / m_{2}=3, N=7$ (четыре импульса у тяжелых и три у легких частиц). Импульсы тяжелых частиц могут иметь значения $p,-p, 2 p$ и $-2 p$. Импульсы легких частиц могут иметь значения $0, p$ и $-p$. В модели возможны три реакции между легкими и тяжелыми частицами, соответствующие ненулевым сечениям $\sigma_{2 p, 0}^{p, p}, \sigma_{-2 p, 0}^{-p,-p}, \sigma_{-p, p}^{p,-p}$. Частицы здесь пронумерованы своими импульсами, причем первые индексы соответствуют тяжелым частицам, а вторые - легким.

Предлагаемая модель имеет прямое обобщение на случай пространства импульсов большей размерности:

Модель 2 (С.А. Амосов, В.В. Веденяпин). $d=2, r=2, m_{1} / m_{2}=3, N=13$ (восемь импульсов у тяжелых и пять у легких частиц). Импульсы тяжелых частиц могут иметь значения $(0, p),(0,-p),(p, 0),(-p, 0),(0,2 p),(0,-2 p),(2 p, 0),(-2 p, 0)$. Легкие частицы имеют импульсы $(0,0),(0, p),(0,-p),(p, 0),(-p, 0)$. В этой модели столкновения такие же, как в модели 1, и симметричные им относительно диагоналей, плюс еще два столкновения бродуэлловского [6] типа между тяжелыми и одно столкновение бродуэлловского типа между легкими частицами (всего $4+2+3=9$ столкновений).

Модель 3 (В.В. Веденяпин, Л. Тоскано). $d=2, r=2, m_{1} / m_{2}=7, N=12$ (шесть импульсов у тяжелых и шесть у легких частиц). Модель симметрична относительно осей, но не симметрична относительно диагоналей. Импульсы частиц имеют следующие координаты: $p_{1}=p_{\overline{1}}=$ $(-1,+1), p_{2}=p_{\overline{2}}=(+1,+1), p_{3}=p_{\overline{3}}=(+1,-1), p_{4}=p_{\overline{4}}=(-1,-1), p_{5}=(0,+1)$, $p_{\overline{5}}=(0,+3), p_{6}=(0,-1), p_{\overline{6}}=(0,-3)$. Мы обозначаем номера легких частиц без черты, а номера тяжелых - с чертой сверху. Столкновениям модели соответствует набор сечений $\sigma_{k, j}^{i, j}$ : одно столкновение между тяжелыми частицами $\sigma_{\overline{4}}^{\overline{3}}, \overline{\frac{1}{2}}$; три столкновения между легкими частицами $\sigma_{6,2}^{5,1}, \sigma_{2,4}^{3,1}, \sigma_{3,6}^{5,4}$; четыре смешанных столкновения $\sigma_{\overline{6}, 5}^{\overline{4}, 1}, \sigma_{\overline{1}, 3}^{\overline{3}, 1}, \sigma_{\overline{1}, 2}^{\overline{2}, 1}, \sigma_{\overline{1}, 4}^{\overline{4}, 1}$.

\section{СПИСОК ЛИТЕРАТУРЫ}

[1] Дирак П. Принципы квантовой механики. М.: Наука, 1979. [2] Mingalev O. V., Orlov Yu. N., Vedenyapin V. V. // Phys. Lett. A. 1996. V. 223. P. 246-250. [3] Веденяпин В. В., Орлов Ю. Н. // Докл. РАН. 1997. Т. 351. №4. С. 444-447. [4] Streater R. Statistical Dynamics. London: Imperial College Press, 1995. [5] Веденяпин В. В., Мингалёв И. В., Мингалёв О. В. // Матем. сб. 1993. Т. 184. №11. С. 21-38. [6] Bobylev A. V., Cercignani C. // J. Statist. Phys. 1998. V. 91. P. 1-2. [7] Vedenyapin V. V. Discrete velocity models for mixtures // Препринт № 17. М.: Ин-т прикл. матем. РАН, 1999.

Институт прикладной математики им. М. В. Келдыша РАН; Неаполитанский университет "Федерико 2" 